\title{
Experimental investigation on the aerodynamic loads and wake flow features of low aspect-ratio triangular prisms at different wind directions
}

\author{
G.V. Iungo*, G. Buresti \\ University of Pisa, Department of Aerospace Engineering, via Caruso 8, 56122 Pisa, Italy
}

Received 9 February 2009; accepted 9 June 2009

Available online 19 August 2009

\begin{abstract}
The results are described of measurements of the mean and fluctuating forces acting on low aspect-ratio triangular prisms placed vertically on a plane, having isosceles triangular cross-section with $60^{\circ}$ or $90^{\circ}$ apex angles and aspect ratios ranging from 1.0 to 3.0. The tests are carried out in a wind-tunnel by varying the wind direction, $\theta$, between $0^{\circ}$ and $180^{\circ}$, at a Reynolds number $\operatorname{Re} \simeq 1.2 \times 10^{5}$. Furthermore, for the model with apex angle of $60^{\circ}$ and aspect ratio 3.0, flow visualizations with tufts and hot-wire measurements are performed, which permit to characterize the wake morphology as a function of wind direction and to assess that an alternate vortex shedding always exists, with a frequency that is roughly inversely proportional to the wake width. The force measurements show that large variations in the mean values of the drag and cross-flow forces occur by varying $\theta$, in strict correspondence with changes in wake flow features. The intensity of the fluctuating cross-flow forces, directly connected with vortex shedding, is found to vary significantly with flow orientation and aspect ratio, and to be approximately proportional to the streamwise projection of the body surface immersed in the separated wake. Finally, an increase in vortex shedding frequency is generally found with decreasing aspect ratio.
\end{abstract}

(C) 2009 Elsevier Ltd. All rights reserved.

Keywords: Bluff body aerodynamics; Aerodynamic forces; Triangular prisms; Wakes

\section{Introduction}

The aerodynamic forces acting on finite-length cylindrical or prismatic bluff bodies placed vertically on a plane and immersed in a cross-flow are strictly dependent on the characteristics of the wakes they produce. In particular, the presence of velocity fluctuations induces fluctuating pressure forces on the bodies, which are a function, in general, of the body cross-section shape, of the body aspect ratio and of the incoming wind direction. Cross-flow force components are present when the average or instantaneous symmetry of the flow is lost, due to the intrinsic geometry of the crosssection or to the presence of alternate vortex shedding.

The main feature characterizing the aerodynamics of finite-length cylinders is the flow passing over the free-end, which strongly affects the wake flow field; in particular, increasingly significant three-dimensionality is introduced with

${ }^{*}$ Corresponding author. Tel.: + 390502217227 ; fax: + 390502217244 .

E-mail address: giacomo.iungo@ing.unipi.it (G.V. Iungo). 
decreasing body aspect ratio, which influences the alternate vortex shedding from the sides of the body, in terms of both frequency and regularity.

Considering the flow around a finite circular cylinder, which is the bluff body to which most investigations were devoted, for large values of the aspect ratio Farivar (1981), Ayoub and Karamcheti (1982) and Fox et al. (1993), showed the presence, for $h / d>10$ (where $h$ is the height and $d$ is the diameter of the cylinder), of a clear alternate vortex shedding from most of the cylinder span, with Strouhal numbers of the same order as those typical of 2-D flow. However, a decrease of the frequency was found in a zone approaching the free-end of the cylinders, probably due to a widening of the wake caused by the flow passing over the free-end and to an increase in the formation length of the shed vortices. The relative extent of this zone increases with decreasing aspect ratio, and for $h / d<5$ the vortex shedding from the whole body takes place at a frequency that is lower than in the corresponding 2-D case [see, e.g. Sakamoto and Arie (1983a)]. Actually, Budair et al. (1991) suggested that alternate vortex shedding disappears when $h / d \leq 7$, but this finding was not confirmed by other investigations, which showed that vortex shedding was still present in the wake generated from shorter cylinders, even if more and more irregular and intermittent with decreasing aspect ratio. Park and Lee (2000) observed that, as the cylinder aspect ratio decreases, the vortex shedding frequency decreases and the vortex formation region increases. Sakamoto and Arie (1983a, 1983b) found a switching from an alternate vortex shedding to a symmetrical shedding of arch-type vortices for a circular cylinder below a critical aspect ratio, i.e. for $h / d \leq 2.5$. In these experiments the two different types of shedding were detected through smoke-wire visualizations, with the bodies immersed in an incoming boundary layer with thickness of the same order as the height of the models. Furthermore, Okamoto and Sunabashiri (1992), through visualizations in a water tunnel, showed that, even with a presumably lower value of the boundary layer thickness, the alternate type of shedding occurred for a circular cylinder for $h / d \geq 4$ and the symmetrical one when $h / d \leq 2$.

Further experimental and numerical results, including also the effect of the incoming boundary layer thickness, are available for circular cylinders of different aspect ratio [see, e.g. Kitagawa et al. (1997, 2002), Park and Lee (2000), Roh and Park (2003), Fröhlich and Rodi (2004), Sumner et al. (2004), Liu et al. (2005), Adamarola et al. (2006), Afgan et al. (2007)]. One of the most interesting features arising from these investigations is the presence of a couple of counterrotating vortices detaching from the free-end, which produce a significant downflow in the inner region of the near wake and an increasing influence on the wake development as the aspect ratio is decreased.

As for the present work, the choice of testing prisms with triangular cross-section was mainly dictated by the small Reynolds number dependence of this geometry (due to the presence of sharp edges fixing the boundary layer separation), and by the possibility of obtaining quite different geometrical and flow conditions by varying the orientation of the models to the wind. Furthermore, very limited data are available in the literature for this body shape and for models placed with different wind directions, as, e.g. Lindsey (1938), El-Sherbiny (1983), Luo et al. (1994), Alonso (2005) and, more recently, Srigrarom and Koh (2008), where a qualitative analysis of the flow generated from a rotationally oscillating equilateral triangular cylinder was recently carried out through flow visualizations and PIV measurements.

In Buresti et al. (1998) and Buresti and Lombardi (2000) the wake flow fields of triangular prisms placed vertically on a plane and having two different isosceles cross-sections (with apex angles of $60^{\circ}$ and $90^{\circ}$ ) were analysed through hotwire measurements; the aspect ratio, $h / w$ (where $w$ is the width of the base side of the triangular cross-sections), was varied between 1.0 and 3.0. By analysing the phase difference between the dominant fluctuations of velocity signals acquired at opposite sides of the wake, they found that alternate vortex shedding occurred for all models, even for an aspect ratio as low as $h / w=1.0$. Two wind directions, $\theta$, were considered, and the alternate vortex shedding from the lateral vertical edges of the prisms with equilateral triangular cross-section was found to be characterized by a Strouhal number $\mathrm{St}=f w / U_{\infty} \simeq 0.1$ when the incoming flow impinged orthogonally to a vertical face of the models $\left(\theta=0^{\circ}\right)$, whereas a dominant frequency corresponding to $\mathrm{St} \simeq 0.16$ was detected when the wind was directed against the apex edge of the prisms $\left(\theta=180^{\circ}\right)$. For this wind direction the reduction in the regularity of the vortex shedding with decreasing aspect ratio was found to be higher than for $\theta=0^{\circ}$.

The higher complexity of the upper near-wake flow field when a prism with triangular cross-section and aspect ratio $h / w=3$ was orientated with its apex edge against the incoming wind was deeply characterized in Iungo and Buresti (2007). Flow fluctuations at three prevailing frequencies were singled out, with different relative intensities depending on the wake regions. In particular, the frequency connected with alternate vortex shedding from the vertical edges of the prism was found to dominate in the regions just outside the lateral boundary of the wake. On the other hand, a lower frequency, at $\mathrm{St} \simeq 0.05$, was found to prevail in the velocity fluctuations on the whole upper wake. Fluctuations with a dominant frequency significantly below that of vortex shedding had already been detected in the upper portions of the wake of finite circular cylinders with higher aspect ratio, e.g. by Kitagawa et al. (1997) and by Park and Lee (2000). Simultaneous measurements carried out over the wake of the prisms at symmetrical locations with respect to the symmetry plane showed that these fluctuations correspond to a vertical, in-phase, oscillation of two counter-rotating 
axial vortices detaching from the front edges of the free-end. This finding was confirmed by the results of an LES simulation of the same flow configuration, described in Camarri et al. (2006), which also highlighted the complex topology of the upper near-wake produced by the vorticity sheets shed from all the edges of the prism. In Iungo and Buresti (2007) wake velocity fluctuations were also observed at an intermediate frequency $\mathrm{St} \simeq 0.09$, and were found to prevail in the symmetry plane. By using the evidence provided by the abovementioned LES simulation, by flow visualizations and by pressure measurements over the prism surface [see also Buresti and Iungo (2008)], it was suggested that they may be caused by a flaglike oscillation of the sheet of transversal vorticity shed from the rear edge of the body free-end, and approximately lying along the downstream boundary of the recirculation region in the central part of the near wake.

The aim of the present work is to better understand the influence of the variation of the wind direction, $\theta$, on the wake flow features and on the mean and fluctuating forces acting on triangular prisms with two different cross-sections and aspect ratio between 1.0 and 3.0. The experimental set-up is described in Section 2. In Section 3, flow visualizations with tufts are presented which were carried out for an equilateral model with aspect ratio $h / w=3.0$, by varying the wind direction from $\theta=0^{\circ}$, i.e. with the free-stream directed orthogonally to a vertical face, to $\theta=60^{\circ}$, i.e. with the incoming flow directed against the apex edge of the prism. These tests allowed the flow features in proximity to the faces of the model to be characterized; in particular, the presence of flow separation bubbles and the position of the reattachment areas were assessed. Subsequently, the results of hot-wire measurements, carried out for the same model in order to estimate the width and position of the wake and to characterize the vortex shedding frequency as a function of wind direction, are reported and discussed in Section 4. In Section 5, force measurements performed on prisms having isosceles triangular cross-sections, with $60^{\circ}$ and $90^{\circ}$ apex angles, are described; the tests were carried out by varying the wind direction from $\theta=0^{\circ}$ up to $180^{\circ}$ and the aspect ratio in the range $1.0 \leq h / w \leq 3.0$. Finally, conclusions are provided in Section 6.

\section{Experimental set-up and procedures}

The tests were carried out in a closed-return subsonic wind tunnel, with circular open test section $1.1 \mathrm{~m}$ in diameter and $1.48 \mathrm{~m}$ in length, and free-stream turbulence level of $0.9 \%$. The general set-up of the tests is shown in Fig. 1 .

Prismatic bodies with two different isosceles triangular cross-sections were used, with apex angles of $60^{\circ}$ (i.e. with equilateral section, denoted as model 60) or $90^{\circ}$ (model 90), and with aspect ratios $h / w=1.0,1.5,2.0$ and 3.0 (where $w=90 \mathrm{~mm}$ ). The models were placed vertically on a horizontal plane, and were connected to a six-component straingage balance supported by a rotatable base placed underneath, so that they could easily be orientated in different wind directions, with the rotation occurring around an axis passing through the centre of gravity of their base section. An NACA 0018 fairing was used to shield the balance and the relevant support. The frame of reference used is shown in Fig. 1; the $x$-axis was chosen in the free-stream direction and the $z$-axis in the vertical direction, positive from the model base to the tip. The $y$ direction was consequently defined, producing a clockwise frame of reference. The analysed flow directions were generally varied in $10^{\circ}$ intervals from $\theta=0^{\circ}$, i.e. with the free-stream directed orthogonally to the larger vertical face, to $\theta=180^{\circ}$, i.e. with the incoming flow directed against the apex edge of the prism. Flow visualizations and hot-wire measurements were performed at a free-stream velocity $U_{\infty}=25 \mathrm{~m} / \mathrm{s}$. Preliminary force measurements for model 60 with $h / w=3.0$ were carried out by varying the velocity in the range of $15-25 \mathrm{~m} / \mathrm{s}$, in order to check the

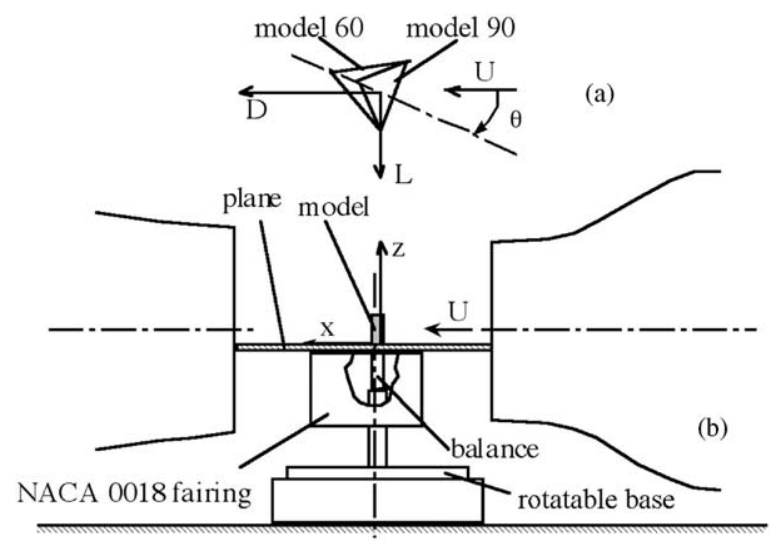

Fig. 1. Experimental layout. 
constancy of the Strouhal number of the spectral peak connected with vortex shedding. Subsequently, all models were tested at a free-stream velocity $U_{\infty}=20 \mathrm{~m} / \mathrm{s}$, corresponding to a Reynolds number $\operatorname{Re}=U_{\infty} w / v \simeq 1.2 \times 10^{5}$. The thickness of the turbulent boundary layer on the plane, $\delta$, was of the order of $15 \mathrm{~mm}$, so that the parameter $h / \delta$ ranged from 6 to 18 .

Velocity measurements were performed with single-component hot-wire anemometry. The used probes were Dantec type 55, connected to an IFA AN 1003 A.A. Lab System test module. A sampling frequency of $2 \mathrm{kHz}$ and a time-length of $33 \mathrm{~s}$ were chosen after extensive preliminary tests. As for the force measurements, the six signals from the balance, each comprising 65536 samples, were simultaneously acquired at a sampling rate of $1 \mathrm{kHz}$, and were processed by means of the balance calibration matrix to obtain the time histories of the load components acting on the models. The maximum errors of the balance measurements were previously estimated to be of the order of $1.5 \%$.

Both the hot-wire and force signals were analysed using conventional Fourier techniques and procedures based on the wavelet and Hilbert transforms. In particular, the complex Morlet wavelet, defined as $\psi(t)=\mathrm{e}^{\mathrm{i} \omega_{0} t} \mathrm{e}^{-t^{2} / 2}$, was used. The spectra described in the following were obtained by integration in time of the wavelet maps obtained by using a central frequency $\omega_{0}=6 \pi$, in order to enhance the frequency resolution. This permits to obtain spectra which are smoother than the Fourier ones and which are mathematically well-defined, without having to use subjective averaging procedures. Finally, in order to extract from the force signals the contributions corresponding to the spectral components associated with vortex shedding, the procedure based on wavelet filtering and Hilbert transform analysis described in Buresti et al. (2004) was used.

\section{Flow visualizations}

Flow visualizations were carried out by positioning tufts on the vertical faces of model 60 with $h / w=3.0$. The objective of these tests was to qualitatively characterize the flow in proximity to the vertical faces of the model for different free-stream directions, $\theta$. The tufts were $10 \mathrm{~mm}$ long and were fixed over the model surface through aluminium tape, with a distance of $5 \mathrm{~mm}$ between two consecutive tufts. All flow visualizations were performed at a free-stream velocity $U_{\infty}=25 \mathrm{~m} / \mathrm{s}$. The free-stream direction was varied from $\theta=0^{\circ}$ up to $60^{\circ}$, because the flow field is symmetric with respect to the wind direction $\theta=60^{\circ}$ in the range of $0^{\circ} \leq \theta \leq 120^{\circ}$, and the configuration replicates after a rotation of $120^{\circ}$ due to the equilateral cross-section of the model.

For the first test series, three horizontal rows of tufts were placed over the leeward vertical face at heights of $z / h=1 / 4,2 / 4$ and $3 / 4$. Starting from $\theta=60^{\circ}$, i.e. with the flow directed against the apex edge of the prism, from the flow visualization reported in Fig. 2(a) it is evident that all the tufts are bent in the downstream direction, indicating a well attached flow on the two lateral vertical surfaces (see sketch in Fig. 3(a)), so that the wake detaches from the two rear vertical edges of the prism. By reducing $\theta$, the flow stagnation area is located on the windward vertical surface of the prism, as sketched in Figs. 3(b) and (c). Therefore, at $\theta=54^{\circ}$ a small separation bubble is already observed in proximity to the front sharp edge of the leeward face, but the larger separation area for $\theta=50^{\circ}$ is shown in Fig. 2(b). In this picture it is seen that several tufts positioned in proximity to the front vertical edge are directed upstream, whereas all the remaining tufts are directed downstream, indicating that a narrow area of separated flow is present near the front vertical edge. At a distance about $10 \mathrm{~mm}(\simeq 0.11 \mathrm{w})$ from the front edge the flow reattaches on the leeward surface and it remains attached as far as the rear vertical edge. The presence of a separation bubble and the consequent flow reattachment on the leeward surface is sketched in Fig. 3(b) for the wind orientation $\theta=50^{\circ}$.

Further reduction of $\theta$ produces a widening of the distance between the model and the shear layer detached from the front vertical edge, so that the flow reattachment on the leeward face takes place more downstream; in fact, for $\theta=44^{\circ}$ it occurs at a distance from the front vertical edge of approximately $45 \mathrm{~mm}(\simeq 0.5 \mathrm{w})$, while for $\theta=38^{\circ}$ the flow reattaches at a distance of $85 \mathrm{~mm}(\simeq 0.94 w)$, as shown in Figs. 2(c) and (d), respectively. With a wind direction corresponding to $\theta=35^{\circ}$ the wake width is increased in such a way that all tufts are found to be directed upstream, as shown in Fig. 2(e), indicating that no reattachment is present anymore on the leeward face (Fig. 3(c)).

From these tests some differences as regards the streamwise location of the flow reattachment on the leeward surface were observed for the three different heights at which the tufts were positioned; thus, a further series of visualizations was performed by setting the tufts on vertical rows at different streamwise positions. The visualizations carried out by fixing the tufts at a distance of $10 \mathrm{~mm}(\simeq 0.11 w)$ from the front vertical edge are first presented. From what was observed through the flow visualization reported in Fig. 2(b), at the wind direction $\theta=50^{\circ}$ this position of the tufts should be roughly coincident with the reattachment area of the flow on the leeward surface; in effect, for this wind direction the tufts are still practically attached over the model surface for the whole height of the model (Fig. 4(a)). Conversely, for $\theta=48^{\circ}$, shown in Fig. 4(b), a separation of the flow occurs, but only in the region with $z / h \leq 2 / 3$; this confirms that the flow reattachment occurs at different streamwise locations along the model height. 

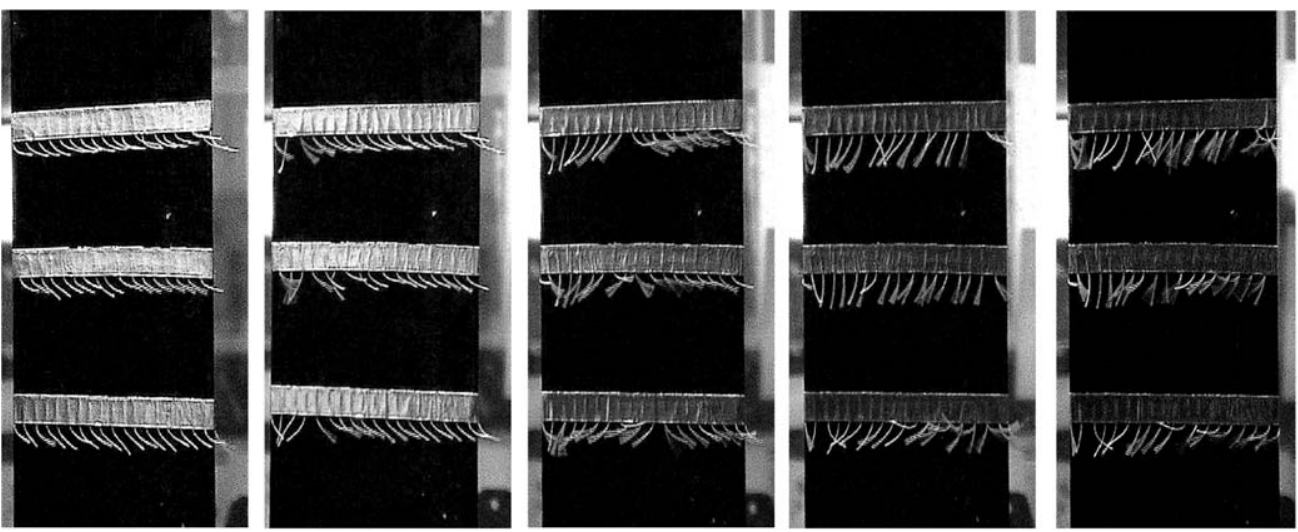

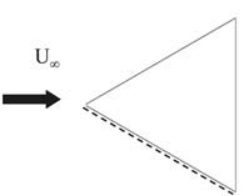

(a)

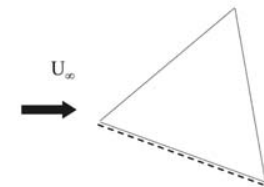

(b)

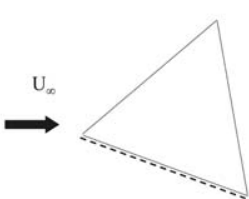

(c)

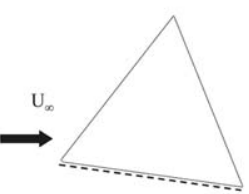

(d)

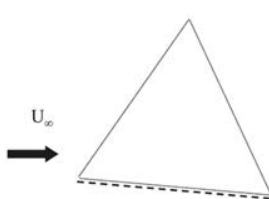

(e)

Fig. 2. Flow visualizations on the leeward vertical face of model 60 with $h / w=3.0$, for different free-stream directions: (a) $\theta=60^{\circ}$; (b) $\theta=50^{\circ}$; (c) $\theta=44^{\circ}$; (d) $\theta=38^{\circ}$ and (e) $\theta=35^{\circ}$. (Wind is directed from left to right and the dashed line in the sketches indicates the face viewed in the images.)

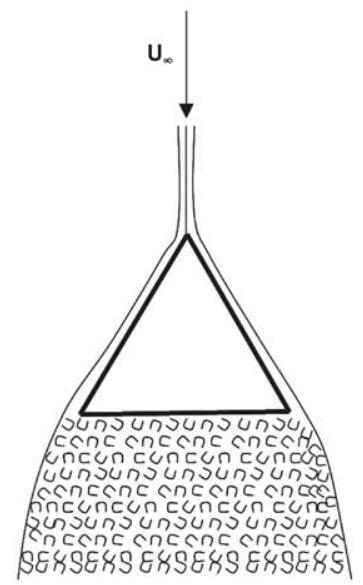

(a)

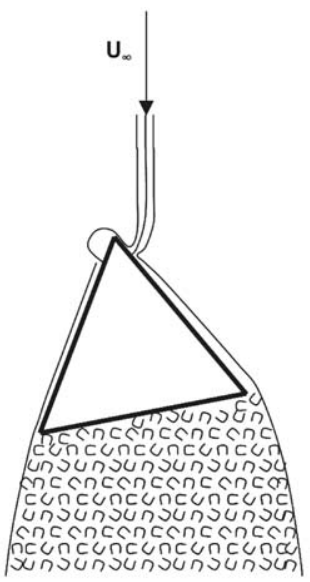

(b)

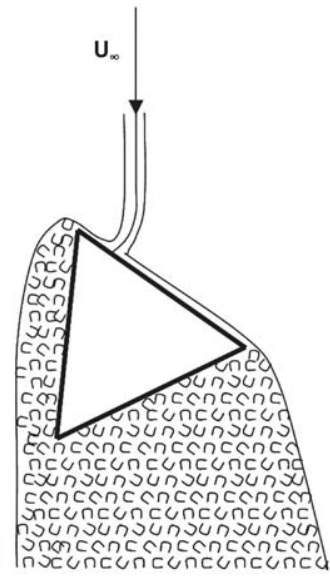

(c)

Fig. 3. Sketches of the flow around the equilateral triangle cross-section for different wind orientations, $\theta$ : (a) $\theta=60^{\circ}$; (b) $\theta=50^{\circ}$ and (c) $\theta=35^{\circ}$.

Setting the tufts more downstream, at a distance of $0.5 w$ from the front edge, an attached flow on the leeward surface is observed for $\theta=44^{\circ}$ (Fig. 4(c)), consistently with the visualization in Fig. 2(c). However, for the wind direction $\theta=42^{\circ}$ (Fig. 4(d)), the flow is generally separated at the location of the tufts, except for two narrow areas in proximity to the model free-end and base. This flow feature is very similar to what observed for a circular cylinder by Pattenden et al. (2005) through surface visualizations; in that case the flow detachment occurring more downstream near the freeend was considered to be the result of the up-wash induced by the axial tip-vortices generated over the model free-end. As already pointed out, the presence of two well-concentrated counter-rotating vortices over the tip of model 60 , for $h / w=3.0$ and $\theta=60^{\circ}$, was also clearly assessed in Iungo and Buresti (2007), and was shown to occur together with significant energy peaks at a Strouhal number $\mathrm{St}=f w / U_{\infty} \simeq 0.05$, in the spectra of velocity signals acquired on the upper wake. The presence of the couple of axial vortices is also confirmed in the present experimental campaign, through the hot-wire measurements described in the next section, for wind orientations ranging from $\theta=40^{\circ}$ to $60^{\circ}$. 

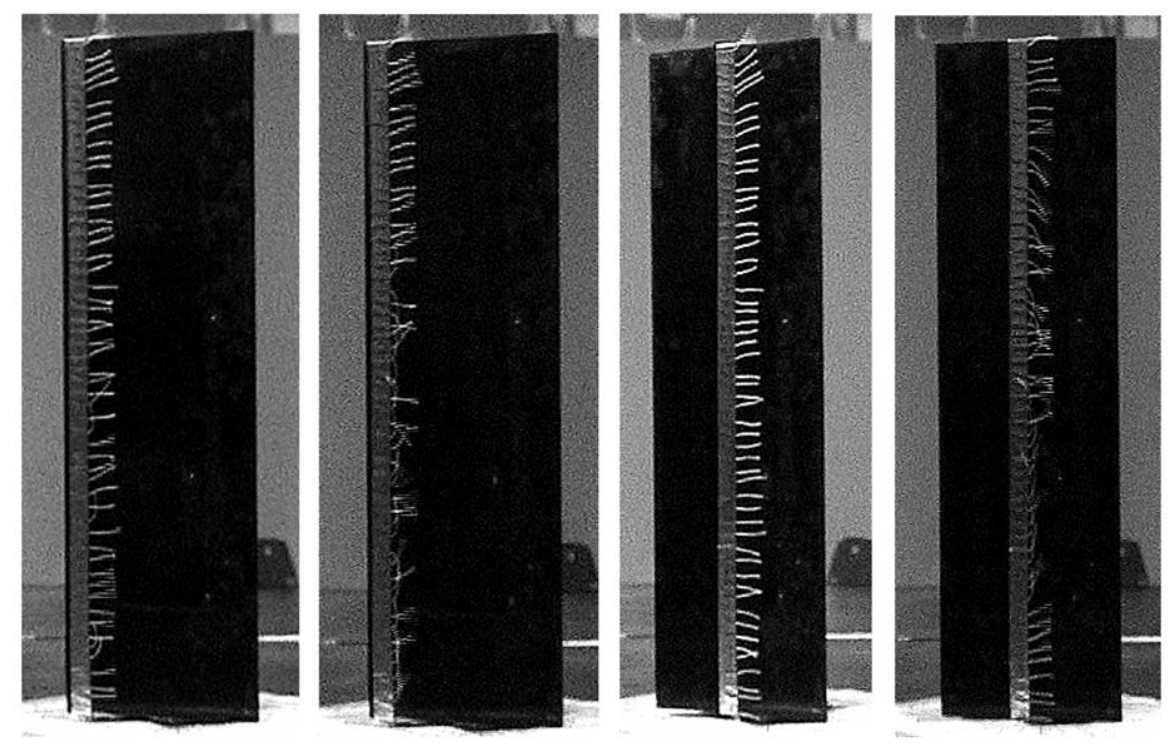

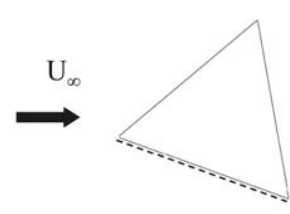

(a)

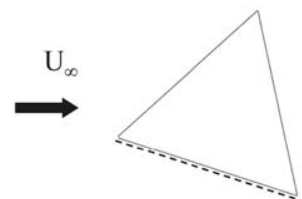

(b)

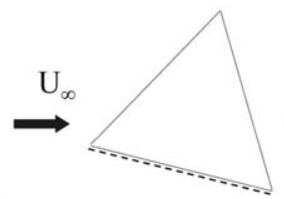

(c)

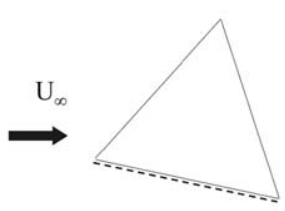

(d)

Fig. 4. Flow visualizations on the leeward vertical face of model 60 with $h / w=3.0$ : (a) $\theta=50^{\circ}$ and tufts at $0.11 w$ from the front edge; (b) $\theta=48^{\circ}$ and tufts at $0.11 \mathrm{w}$ from the front edge; (c) $\theta=44^{\circ}$ and tufts at $0.5 \mathrm{w}$ from the front edge and (d) $\theta=42^{\circ}$ and tufts at $0.5 \mathrm{w}$ from the front edge. (Wind is directed from left to right and the dashed line in the sketches indicates the face viewed in the images.)

Therefore, the premature flow reattachment on the leeward face of the prism in proximity to the model free-end may reasonably be ascribed to the up-wash generated by the tip-vortices, analogously to what already observed for the circular cylinder. Regarding the wake reattachment in proximity to the model base, in analogy to what found by Pattenden et al. (2005), it might be explained as the result of the flow field generated by the horseshoe vortex present in this region; however, in the present experiments no deeper investigations were carried out to check the possible presence of a significant horseshoe vortex in this area.

\section{Hot-wire measurements}

Hot-wire measurements were carried out for model 60 with $h / w=3.0$, in order to characterize the width and position of the wake by varying the wind direction in the range $0^{\circ} \leq \theta \leq 60^{\circ}$. Moreover, the dominating spectral components of the velocity field were detected for each wind direction. For these tests the origin of the $x$-axis is assumed to be on the base plane, at the central point of the rear face of the model positioned at $\theta=60^{\circ}$. As suggested in Iungo and Buresti (2007), traverses were performed at $x / w=2.5$ and $z / h=0.3$, a wake region where the spectral component connected with vortex shedding typically prevails. Tests were performed for $-2.5 \leq y / w \leq 2.5$, with a space-step of $0.125 w$. An example of the results of the measurements, corresponding to the wind direction $\theta=33^{\circ}$, is shown in Fig. 5, where the mean value, standard deviation and skewness of the velocity signals are reported; the data show the typical trends encountered in a traverse of the wake.

The characterization of a representative width of the wake from data like those of Fig. 5 is not immediate, and must inevitably be based on a conventional definition. Different choices may be made for the purpose, the most meaningful ones being the distances between the two peaks of the standard deviation, between the zero crossings of the skewness, and between the two negative peaks of the skewness. The first two choices give similar values, corresponding approximately to the distance between the zones of maximum absolute value of the derivative of the mean velocity 


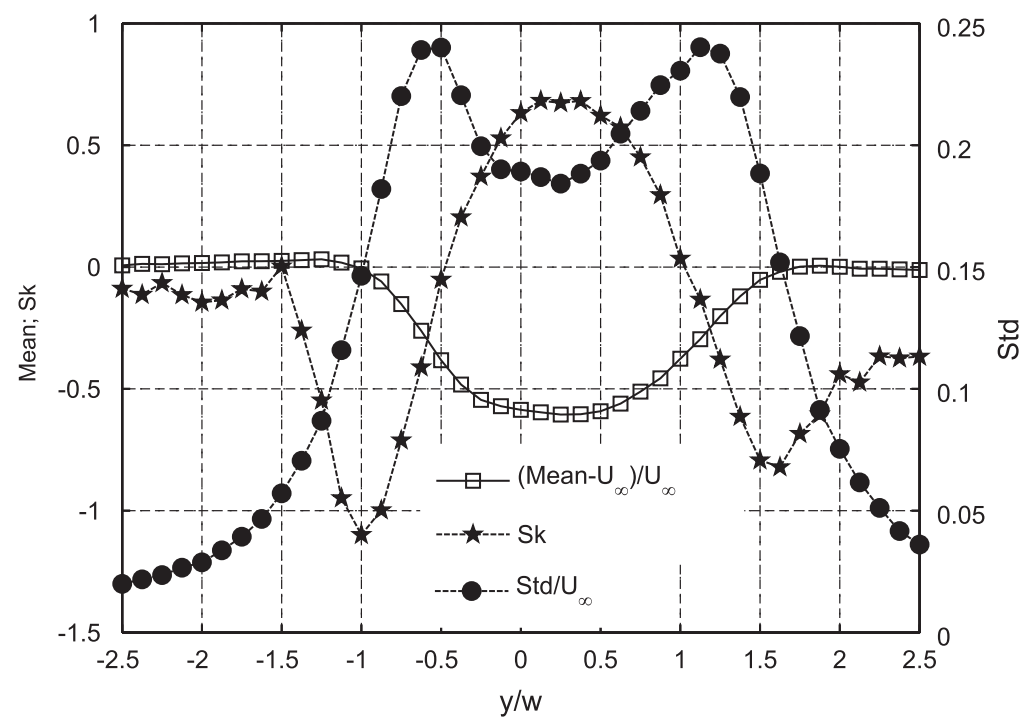

Fig. 5. Statistics of the hot-wire measurements carried out for the wind direction $\theta=33^{\circ}$ at $x / w=2.5$ and $z / h=0.3$.

(which, however, is more difficult to characterize), while the last one provides a larger value, more connected with the distance between the opposite regions where the mean velocity has just started decreasing. In the present paper the distance between the peaks in the standard deviation curve has been adopted as a representative reference value of the wake cross-flow width, and is denoted as $w_{w}$.

Starting from the configuration $\theta=0^{\circ}$, and increasing the wind direction angle up to $\theta=40^{\circ}$, it is seen in Fig. 6(a) that the lateral boundaries of the wake move consistently with the displacements of the front vertical edges of the prism (see sketches in Fig. 6(b)). In particular, the standard deviation peak located at a negative $y$ coordinate moves more rapidly than the one at a positive $y$ coordinate. However, a more significant narrowing of the wake and a decrease in the peak standard deviation values is observed by increasing $\theta$ from $35^{\circ}$ to $40^{\circ}$, witnessing the reattachment of the flow on the leeward surface placed at a negative $y$ coordinate, in agreement with the evidence from the flow visualizations.

Subsequently, for $\theta$ varying from $40^{\circ}$ to $60^{\circ}$ (see Fig. 7(a)), even if for $\theta<60^{\circ}$ a flow separation bubble is generally present on the leeward surface, the flow detachment occurs at the rear vertical edges and the wake boundaries closely follow their positions, as sketched in Fig. 7(b).

The spectral analysis of the velocity signals shows the presence of a dominating component for each tested wind direction; as discussed in detail in Buresti et al. (1998), Buresti and Lombardi (2000) and Iungo and Buresti (2007), this spectral peak can confidently be attributed to alternate vortex shedding from the vertical edges of the model. In Fig. 8(a), examples of the wavelet spectra of the velocity signals acquired at a cross-distance of $1 w$ from the wake boundary are shown. In this figure a reduction of the intensity of the fluctuation energy connected with vortex shedding can be observed with increasing $\theta$; in effect, the wavelet spectra related to the configurations with $\theta=50^{\circ}$ and $60^{\circ}$ are not reported because their fluctuation energy is one order of magnitude smaller than for the remaining wind directions. This feature might be due to a reduction, with increasing $\theta$, of the strength of the shed vortices, connected with a decrease of the velocity in correspondence to the vertical edges of the model where flow separation occurs. As a matter of fact, the instantaneous flux of vorticity from a separating boundary layer is proportional to the square of the velocity at its outer boundary. However, a perhaps more important reason may be that, as discussed in the following, for these wind directions the two counter-rotating axial vortices generated over the free-end produce a higher threedimensionality of the vortices shed from the lateral edges and an increase in their formation length.

From the peaks in the wavelet spectra, the variation of the Strouhal numbers corresponding to vortex shedding was then obtained as a function of wind direction. This variation is shown in Fig. 8(b), together with the curves giving the trends of the geometrical cross-flow width of the model, $w^{*}$, and of the cross-flow width of the wake, $w_{w}$. In the figure the variation of the Strouhal number is described through the parameter $\mathrm{St}\left(\theta=0^{\circ}\right) / \mathrm{St}$, whereas $w^{*}$ is expressed in terms of ratio with the width of the cross-section base side, $w^{*} / w$, and $w_{w}$ is reported as the ratio with the respective value evaluated for the wind direction $\theta=0^{\circ}$, i.e. $w_{w} / w_{w}\left(\theta=0^{\circ}\right)$. 


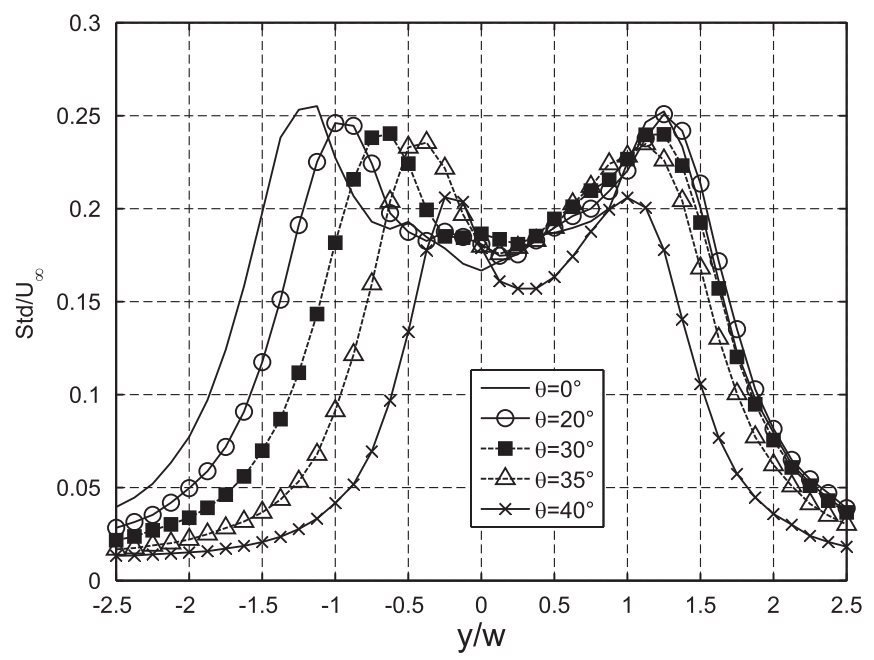

(a)

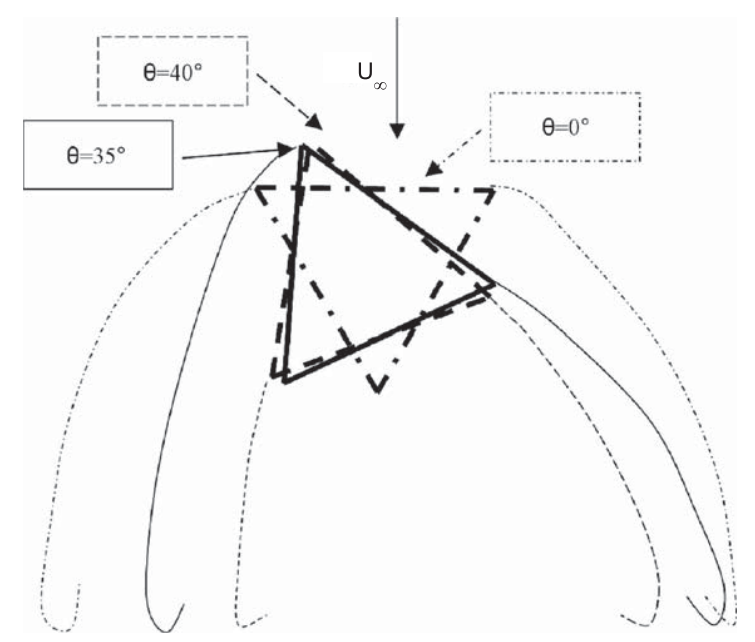

(b)

Fig. 6. Wake characterization: (a) standard deviation of the hot-wire measurements carried out at $x / w=2.5$ and $z / h=0.3$ for wind orientations between $\theta=0^{\circ}$ and $40^{\circ}$ and (b) sketches of the wake for wind orientations between $\theta=0^{\circ}$ and $40^{\circ}$.

As can be seen, the trend of $\operatorname{St}\left(\theta=0^{\circ}\right) / \mathrm{St}$ closely follows the one describing the wake width variation, $w_{w} / w_{w}\left(\theta=0^{\circ}\right)$, up to $\theta=35^{\circ}$. This is not a surprise, as the Strouhal number is expected to be inversely proportional to the wake crossflow width. However, for larger values of $\theta$ the parameter $\operatorname{St}\left(\theta=0^{\circ}\right) / \mathrm{St}$ is higher than $w_{w} / w_{w}\left(\theta=0^{\circ}\right)$, suggesting that the Strouhal number is lower than what could be expected from the reduction of the wake width. This variation of the connection between Strouhal number and wake cross-flow width may perhaps be due to the significant changes that occur in the wake structure by varying the wind direction. A first indication of these changes may be obtained by following the differences and similarities between the trends of $w_{w}$ and $w^{*}$. As a matter of fact, for $\theta$ varying from $0^{\circ}$ to $30^{\circ}$ the flow is completely separated over the lateral surfaces, and thus the wake width, $w_{w}$, decreases following the decrease of the model cross-flow width, $w^{*}$. For $\theta \geq 30^{\circ}, w^{*}$ inverts its trend, i.e. it grows with increasing $\theta$, whereas $w_{w}$ continues to decrease up to $\theta=40^{\circ}$. The minimum in $w_{w}$ is probably due to the fact that, as was observed from the flow visualizations and from the hot-wire statistics, around $\theta=40^{\circ}$ the flow reattaches on the leeward face. For larger values of $\theta\left(40^{\circ} \leq \theta \leq 60^{\circ}\right)$, the wake detaches from the two rear vertical edges of the model, and thus $w_{w}$ grows following the increase of $w^{*}$.

Now, the reattachment of the flow significantly alters the structure of the wake, because it approximately corresponds to the appearance of the two concentrated counter-rotating vortices over the prism free-end. This was also ascertained 


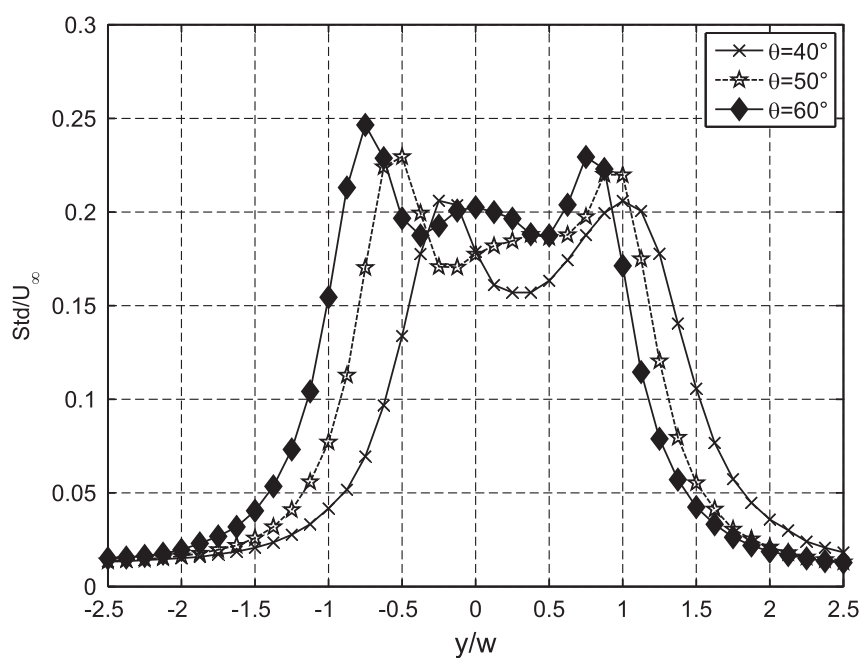

(a)

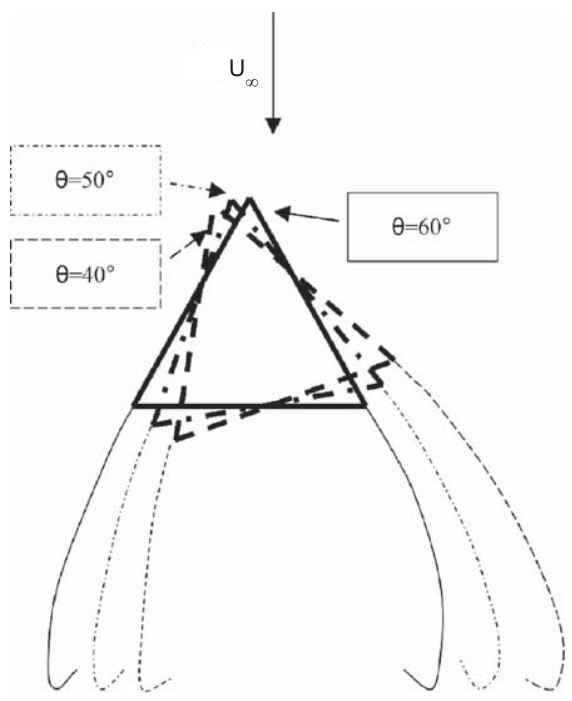

(b)

Fig. 7. Wake characterization: (a) standard deviation of the hot-wire measurements carried out at $x / w=2.5$ and $z / h=0.3$ for wind orientations between $\theta=40^{\circ}$ and $60^{\circ}$ and (b) sketches of the wake for wind orientations between $\theta=40^{\circ}$ and $60^{\circ}$.

by the presence of a low-frequency component in the spectra of the hot-wire signals, which dominates in the upper part of the wake, and remains constant at $\mathrm{St} \simeq 0.05$ even if, as shown in Fig. 8(a), the spectral component connected with vortex shedding varies its frequency with varying $\theta$. In Iungo and Buresti (2007) it was shown that this component may be ascribed to a vertical, in-phase, oscillation of the couple of axial vortices. Therefore, it is not unreasonable to infer that the structure of the wake be significantly changed by this variation of the free-end flow. In effect, the axial vortices produce a larger downwash in the upper near-wake, and the flow three-dimensionality is increased, with larger differences in the vertical direction. In particular, the wake width increases in the downward direction, and this may affect the alternate vortex shedding from the lateral edges, producing also an increase in the formation length of the vortices. In summary, it is probable that the characteristic wake width to which the Strouhal number scales may not be obtained, as was done for the evaluation of $w_{w}$, by referring to a constant downstream position in the wake. The results obtained might indeed be explained by assuming that for $\theta \geq 40^{\circ}$ the characteristic wake width for vortex shedding is actually larger than the measured $w_{w}$. 


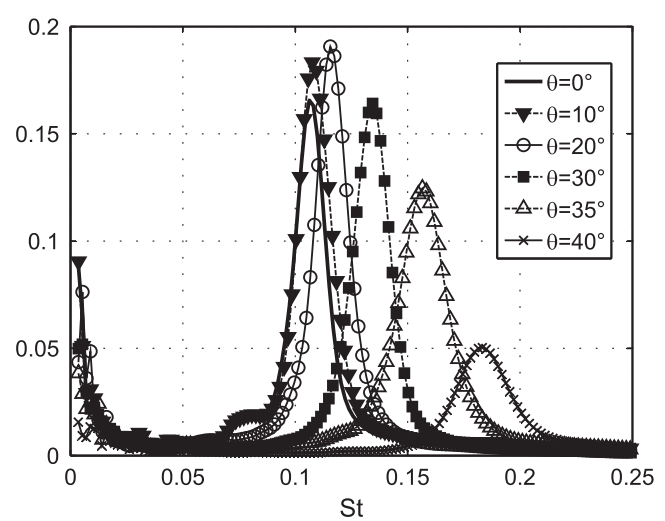

(a)

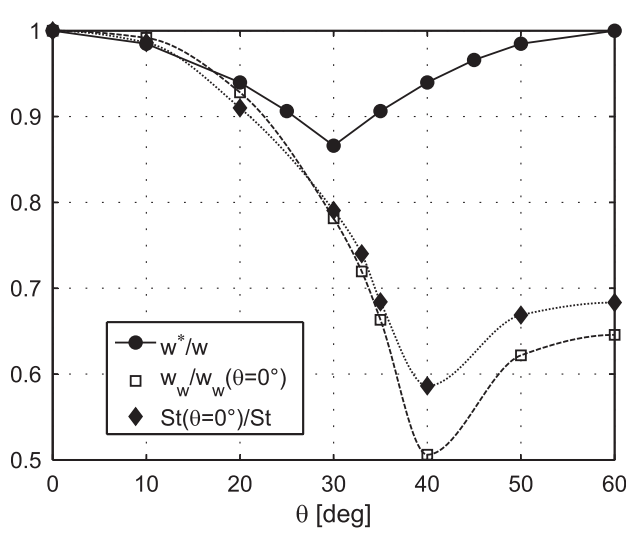

(b)

Fig. 8. Spectral analysis of the hot-wire measurements: (a) wavelet spectra evaluated for the velocity signals acquired at a crossdistance of $1 w$ from the wake boundary and with different wind directions and (b) comparison of the model cross-flow width, $w^{*}$, wake cross-flow width, $w_{w}$, and vortex shedding frequency, $\mathrm{St}$, for different wind directions, $\theta$.

\section{Force measurements}

\subsection{Mean forces}

The variations with wind direction, $\theta$, and aspect ratio, $h / w$, of the mean drag and cross-flow force coefficients, $C_{D}$ and $C_{L}$, are shown in Fig. 9 for model 60, while the analogous data for model 90 are given in Fig. 10. The reference surface for the coefficients in these figures is $h w$. As can be seen, the absolute values of the force coefficients tend to slightly decrease with decreasing aspect ratio due to the three-dimensionality of the flow generated by the body free-end; however, this trend is not a remarkable one.

Considering the two extreme flow orientations, for $\theta=0^{\circ}$ the two model types are characterized by a different streamwise extension of the afterbody (i.e. of the portion of body surface immersed in the separated wake), while they are identical as regards the forebody (which is a rectangular flat surface orthogonal to the free-stream). Conversely, for $\theta=180^{\circ}$ (or $\theta=60^{\circ}$ for model 60 ) the forebodies are different and the afterbody is the same. For these two orientations the corresponding drag coefficients for the 2-D case are available from E.S.D.U. (1979), and are reported for comparison in Figs. 9(a) and 10(a). On the other hand, for the 2-D case the available data on the variation of the forces with wind direction for the models considered in the present investigation are very limited. Actually, to the authors' knowledge the only experimental investigation regarding the effect of wind orientation for cylinders with isosceles triangular cross-sections with various apex angles is described in Alonso (2005). In that work the mean force coefficients were evaluated by using as reference area the product of the model span and the maximum characteristic length of the cross-section of the prisms, which for the present models coincides with $w$, and the relevant data are also shown in Figs. 9 and 10 .

A point that is apparent from these figures is that, for the wind directions for which the comparison is possible, the 2D values reported by Alonso (2005) are generally lower than those given by E.S.D.U. (1979). Particularly puzzling are the results for $\theta=0^{\circ}$, for which equal values are given for both models in E.S.D.U. (1979) and also similar ones are found in the present investigation, whereas considerably different values are reported by Alonso (2005). It must also be pointed out that in the tests described in the latter work the forces on a triangular cylinder with apex angle of $30^{\circ}$ were also measured as a function of $\theta$; however, even for that case, the reported values are significantly lower, for all wind directions, than those obtained by Lindsey (1938) for a similar cylinder. The origin of these discrepancies is not clear, even if experimental uncertainties, connected, for instance, with the reference velocity or with the value of the blockage ratio [which in Alonso (2005) was up to $12.5 \%$, and varied with wind orientation] might have an influence on the obtained data.

On the other hand, the qualitative trends as a function of wind direction of the force coefficients reported in Alonso (2005) are analogous to the respective ones obtained from the present experimental campaign. Nonetheless, the quantitative differences (which are expected as a result of the flow three-dimensionality of the present cases) are not of the same order for all wind orientations. In particular, the differences are much smaller for certain ranges of $\theta$, viz. $30^{\circ} \leq \theta \leq 35^{\circ}$ and $80^{\circ} \leq \theta \leq 85^{\circ}$ for model $60,70^{\circ} \leq \theta \leq 100^{\circ}$ and $140^{\circ} \leq \theta \leq 150^{\circ}$ for model 90 . 


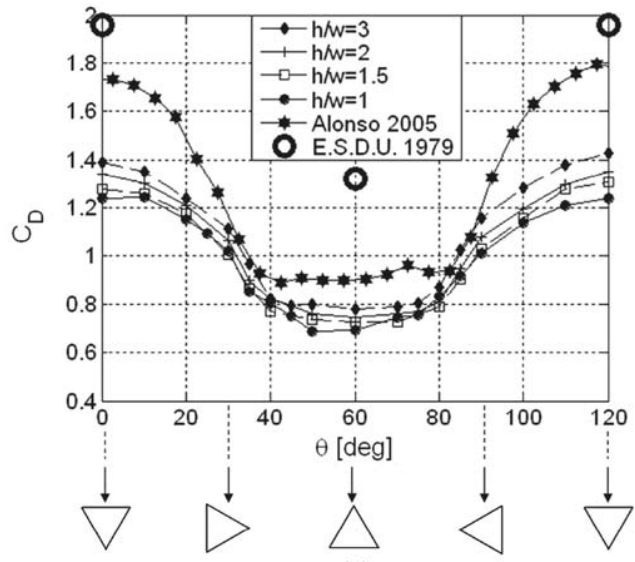

(a)

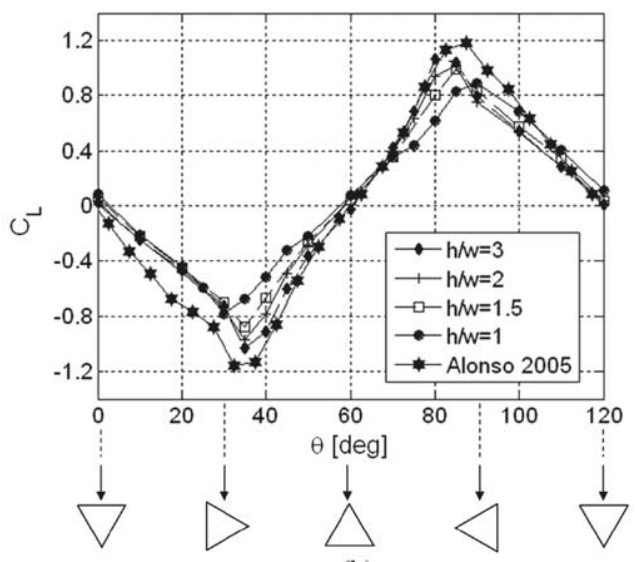

(b)

Fig. 9. Mean forces acting on model 60 with different aspect ratios, as a function of wind direction, $\theta$ : (a) mean drag coefficient, $C_{D}$ and (b) mean cross-flow force coefficient, $C_{L}$.

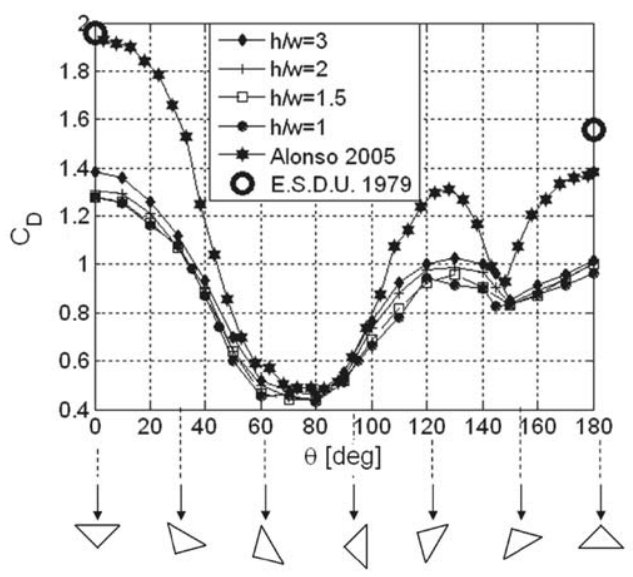

(a)

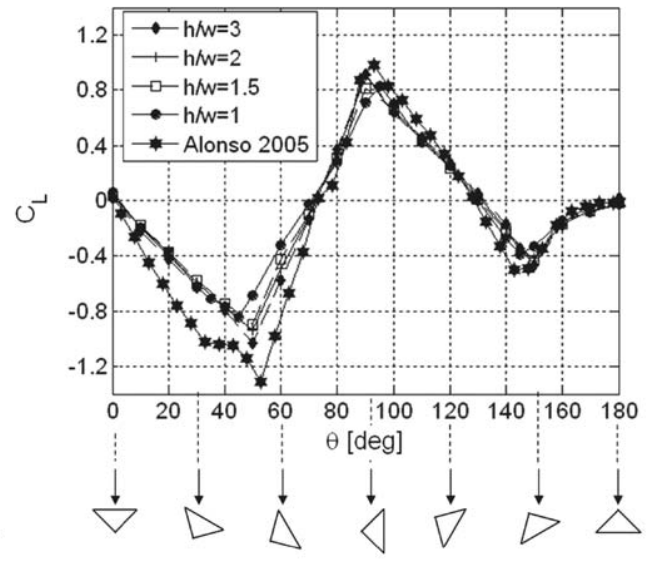

(b)

Fig. 10. Mean forces acting on model 90 with different aspect ratios, as a function of wind direction, $\theta$ : (a) mean drag coefficient, $C_{D}$ and (b) mean cross-flow force coefficient, $C_{L}$.

As regards a detailed analysis of the present results, it is seen that for $\theta=0^{\circ}$ both model 60 and model 90 are characterized by almost the same drag coefficient, but a certain decrease is found with decreasing aspect ratio. Conversely, when the models are placed with their apex edge against the incoming flow, a lower drag is found for model 60, as expected. An interesting point is that the ratios between the present values and the 2-D ones given in E.S.D.U. (1979) show non-negligible differences for the various models and wind orientations. For instance, for $h / w=3.0$ the ratios are approximately $0.71,0.65$ and 0.6 , respectively for both models at $\theta=0^{\circ}$, for model 90 at $\theta=180^{\circ}$ and for model 60 at $\theta=180^{\circ}$ (or $\theta=60^{\circ}$ ). These differences might be explained with the different behaviour of the flow rounding the free-end of the models in the various configurations.

Let us now consider in more detail the trends obtained for model 60 (Fig. 9). Starting from $\theta=0^{\circ}$, i.e. with a vertical face set normal to the wind direction, the flow is separated on the two lateral faces (as already sketched in Fig. 7(b)), so that with increasing $\theta$ a monotone reduction of the mean drag coefficient, $C_{D}$, is found, consistently with the reduction of the wake cross-flow width, $w_{w}$, as assessed in Fig. 8(b) from the hot-wire measurements. Subsequently, between $\theta=35^{\circ}$ and $\theta=40^{\circ}$ a reattachment of the flow on the leeward surface has been detected from the flow visualizations (Section 3) and the hot-wire measurements (Section 4), and a negative peak of the cross-flow force is observed in Fig. 9(b). By further increasing $\theta\left(40^{\circ} \leq \theta \leq 60^{\circ}\right)$, the wake detaches from the two rear vertical edges and a widening of 
the wake has been observed from the hot-wire measurements, whereas a limited further reduction of $C_{D}$ is found. This different behaviour may be ascribed to the significant change of the structure of the wake flow occurring when model 60 is oriented with $40^{\circ} \leq \theta \leq 60^{\circ}$. As a matter of fact, the presence of the couple of counter-rotating vortices detaching from the front edges of the free-end produces a significant downwash flow, which interacts with the wake and with the shedding of vortices from the sides of the models; the result is a more 3-D wake, as already highlighted by the flow visualizations reported in Iungo and Buresti (2007) and by the LES simulation presented in Camarri et al. (2006) for the prism with $h / w=3.0$ and $\theta=60^{\circ}$. Furthermore, the downwash caused by the axial vortices is probably strictly linked to the strong recirculating flow whichwas found to be present in the near wake for the same case; as shown by Buresti and Iungo (2008), this flow gives rise to an increase in the pressure acting over the lower part of the rear face of the prism, which would explain a lower drag coefficient than would be expected from the variation of the width of the wake.

For wind directions $60^{\circ} \leq \theta \leq 120^{\circ}$, the trend of $C_{D}$ is symmetric due to the equilateral cross-section of model 60 , so that $C_{D}$ increases with a monotone trend, while the cross-flow force coefficient, $C_{L}$, is antisymmetric and reaches its maximum value around $\theta \simeq 85^{\circ}$, i.e. when the flow becomes again completely separated from the leeward surface.

As for the variation of the cross-flow force coefficient, $C_{L}$, reported in Fig. 9(b), it can also be observed that the peak values, corresponding to the flow separation from the leeward surface, occur for slightly different wind directions by varying the model aspect ratio, $h / w$. In effect, starting from $\theta=60^{\circ}$ and increasing $\theta$, the maximum value of $C_{L}$ occurs at approximately $\theta=85^{\circ}$ for the model with $h / w=3.0$, whereas for $h / w=1.0$ it occurs, with a smaller absolute value, at $\theta=90^{\circ}$. Analogously, when $\theta$ is decreased, the negative peak is found at $\theta=35^{\circ}$ for $h / w=3.0$ and at $\theta=30^{\circ}$ for $h / w=1.0$. Therefore, the slope of the lift coefficient, $C_{L}$, decreases with reducing aspect ratio. This behaviour can be ascribed to the flow reattachment occurring in proximity to the model tip due to the up-wash generated by the tip vortices, already highlighted from the flow visualizations presented in Section 3. As expected, this effect becomes more and more important as the model aspect ratio is decreased.

For model 90 no flow visualizations or velocity measurements were carried out in the present investigation, so that a characterization of the wake morphology can be performed only through a synergic analysis, for the different wind directions, of the force coefficients, reported in Figs. 10(a) and 10(b), and of the model cross-flow width, $w^{*} / w$. Moreover, the wake features can also be inferred from an analysis of the detailed wall pressure measurements described by El-Sherbiny (1983) for a 2-D triangular cylinder with a $90^{\circ}$ apex angle.

Starting from the wind direction $\theta=0^{\circ}$, i.e. with the largest vertical face set normal to the wind direction, the flow stagnation point is located on this face in correspondence to the symmetry plane and the flow is separated over the two lateral faces. With increasing $\theta$, from the pressure measurements reported in El-Sherbiny (1983) the stagnation point is seen to move towards the front vertical edge; consistently, a monotone reduction of $C_{D}$ is found in the present results, following the decrease of the model cross-flow width, while the modulus of $C_{L}$ increases due to the loss of the flow symmetry.

From the abovementioned pressure measurements, it can be deduced that in the 2-D case a flow reattachment on the leeward face occurs at $\theta=55^{\circ}$, which is consistent with the peak of $C_{L}$ observed around $\theta=50^{\circ}$ from the present experimental campaign (the force values for $\theta=55^{\circ}$ are not available because an angular step of $10^{\circ}$ was used) and with the analogous one also found at $\theta=55^{\circ}$ by Alonso (2005). A sketch of the wake generated for $\theta=50^{\circ}$ is reported in Fig. 11(a). For the model with aspect ratio $h / w=1.0$ the peak of the cross-flow force coefficient is observed at $\theta=45^{\circ}$, indicating that the flow reattachment on the leeward surface occurs for smaller values of $\theta$ with decreasing aspect ratio; as already mentioned for model 60 , this feature may be due to the increased influence of the up-wash generated from the axial vorticity structures present in proximity to the model free-end.

From El-Sherbiny (1983), it may also be seen that the reattachment point on the leeward face moves upstream with increasing $\theta$, up to $\theta=67.5^{\circ}$, at which a completely attached flow over the two lateral faces is present. This particular wind direction corresponds to the line bisecting the cross-section angle set against the incoming flow, as sketched in Fig. 11(b), and a negligible cross-flow force is indeed detected in the present experimental campaign for the nearest available wind direction, i.e. $\theta=70^{\circ}$. The region around this wind direction corresponds to a plateau where the minimum drag coefficients for this model are found.

In the 2-D case, for $\theta>67.5^{\circ}$ the stagnation point shifts to the lateral face and the largest vertical face becomes the leeward face. By increasing $\theta$, a separation bubble is detected on the leeward surface and its reattachment point moves downstream. At $\theta=90^{\circ}$ the flow is again completely separated on the leeward surface (see sketch in Fig. 11(c)), and this wind direction corresponds to a peak of the cross-flow force coefficient.

The drag coefficient is found to increase for $\theta>67.5^{\circ}$ up to $\theta \simeq 120^{\circ}$, where a local maximum is observed. For higher values of $\theta, C_{D}$ decreases and reaches a local minimum at $\theta=150^{\circ}$. From the 2-D pressure measurements, at $\theta=150^{\circ}$ the flow is found to reattach on the leeward face, as sketched in Fig. 11(d), and a local negative peak of the cross-flow coefficient is observed in Fig. 10(b). For $\theta>150^{\circ}$ the reattachment point of the separation bubble on the leeward surface moves upstream, and at $\theta=180^{\circ}$ the flow is completely attached over the two lateral faces. For the range 


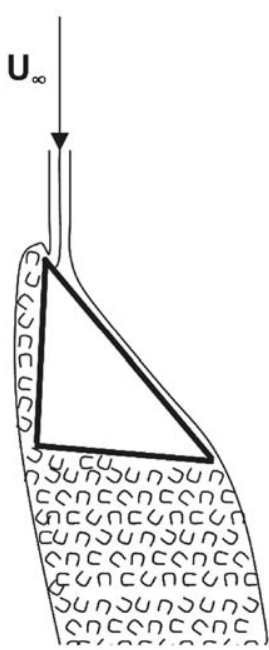

(a)

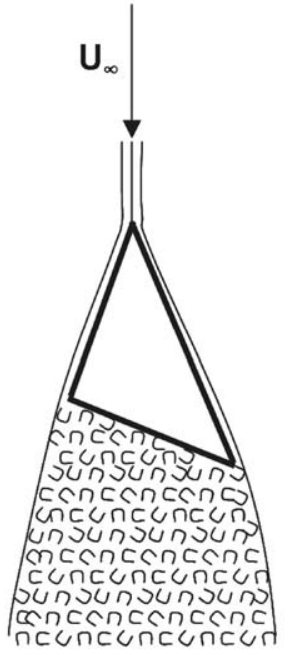

(b)

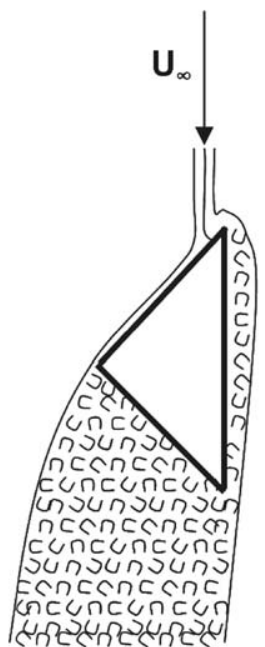

(c)

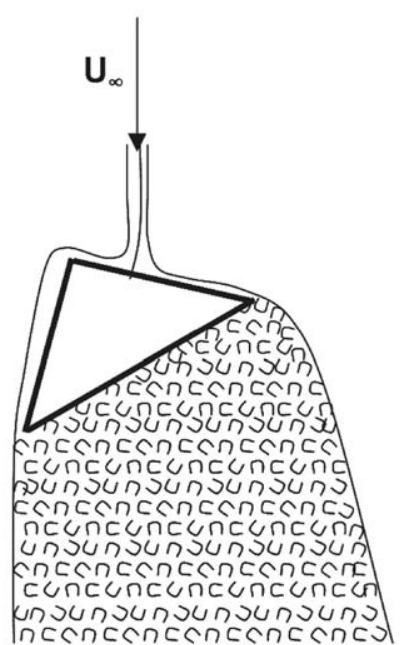

(d)

Fig. 11. Sketches of the wake generated from model 90 set with different wind directions: (a) $\theta=50^{\circ}$; (b) $\theta=67.5^{\circ}$; (c) $\theta=90^{\circ}$ and (d) $\theta=150^{\circ}$.

$150^{\circ} \leq \theta \leq 180^{\circ}$, the wake always detaches from the two rear vertical edges and $C_{D}$ slightly increases, probably due to the widening of the wake width.

Analogously to what already observed for model 60, in Fig. 10(b) a reduction of the slope of $C_{L}$ with decreasing aspect ratio can also be observed for model 90 , for wind directions at which the wake detaches from the rear vertical edges (i.e. $50^{\circ} \leq \theta \leq 90^{\circ}$ ).

\subsection{Fluctuating forces}

The objective of these tests was to ascertain if the vortex shedding could produce significant fluctuating forces on the different models, and to describe their possible variation as a function of wind direction and model aspect ratio.

The first series of tests was carried out on model 60 with aspect ratio $h / w=3.0$, by varying the wind direction in the range $0^{\circ} \leq \theta \leq 120^{\circ}$. Wavelet spectra of the cross-flow force measured at $U_{\infty}=20 \mathrm{~m} / \mathrm{s}$ and different wind directions are shown in Fig. 12(a). In these spectra the high energy content at low frequencies, i.e. below $\mathrm{St}=0.025$, is probably connected with the wind tunnel free-stream turbulence, which is indeed characterized by an energy concentration at those low frequencies. On the other hand, the peaks in the range $0.1 \leq \mathrm{St} \leq 0.2$ may confidently be associated with the alternate vortex shedding.

For this model, even if vortex shedding exists for all wind directions (as assessed from the hot-wire measurements presented in Section 4), a dominant spectral component in the wavelet spectra of the cross-flow force could clearly be detected only for wind directions for which a significant streamwise extent of the afterbody, i.e. of the portion of body surface immersed in the separated wake, is present $\left(0^{\circ} \leq \theta \leq 40^{\circ}\right.$ and $\left.80^{\circ} \leq \theta \leq 120^{\circ}\right)$. From the wavelet spectra reported in Fig. 12(a), a decreased fluctuation energy peak is observed for $\theta=40^{\circ}$ with respect to the ones detected for the remaining wind directions. This feature may be ascribed to the previously assessed flow reattachment on the leeward surface occurring in the range $35^{\circ} \leq \theta \leq 40^{\circ}$.

In Fig. 12(b) the Strouhal numbers derived from the peaks in the cross-flow force spectra are compared with those obtained from the hot-wire measurements for $U_{\infty}=25 \mathrm{~m} / \mathrm{s}, x / w=2.5$ and $z / h=0.3$. In this figure the force-related values corresponding to three different free-stream velocities, viz. 15,20 and $25 \mathrm{~m} / \mathrm{s}$, are also shown; in effect, tests at three different velocities were carried out for this model in order to check the constancy of the Strouhal number corresponding to the spectral peaks. As can be appreciated from Fig. 12(b), the values of St obtained are practically independent from $U_{\infty}$ and coincide with the hot-wire data, which is a further confirmation of the association between the fluctuating cross-flow forces and the alternate vortex shedding from the model. The remaining force measurements for all the models were then carried out for $U_{\infty}=20 \mathrm{~m} / \mathrm{s}$.

It must be pointed out that the drag spectra generally did not exhibit any dominating frequency, except for wind directions corresponding to a flow condition in which the shear layer is nearly reattaching on the leeward vertical 


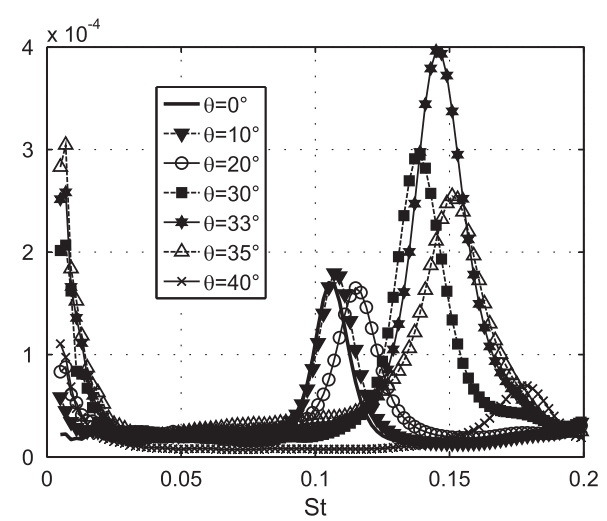

(a)

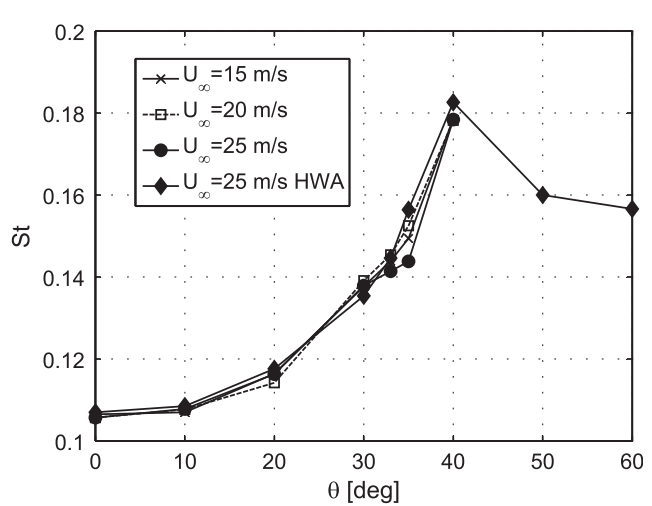

(b)

Fig. 12. Spectral characterization of cross-flow force for model 60 with $h / w=3.0$ : (a) wavelet spectra measured at $U_{\infty}=20 \mathrm{~m} / \mathrm{s}$ and different wind directions and (b) Strouhal number connected with vortex shedding and comparison with the results obtained from the hot-wire measurements (HWA) as a function of the wind direction.

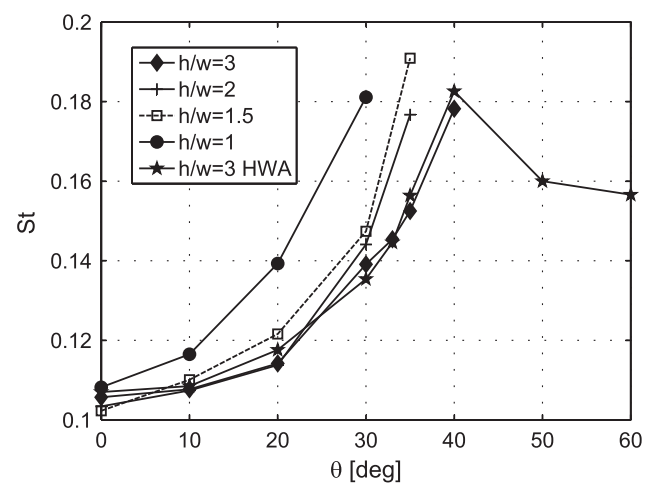

(a)

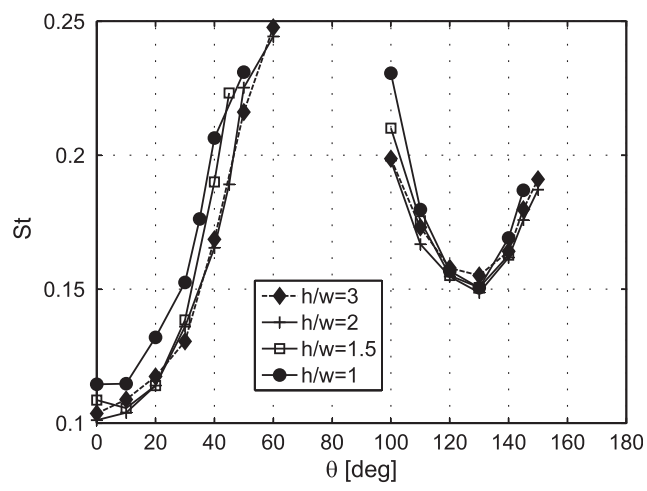

(b)

Fig. 13. Strouhal numbers connected with vortex shedding, evaluated from the cross-flow force signals, for models with different crosssections and aspect ratios: (a) model 60 and hot-wire measurements (HWA) and (b) model 90.

surface of model $60\left(30^{\circ} \leq \theta \leq 40^{\circ}\right.$ and $\left.80^{\circ} \leq \theta \leq 90^{\circ}\right)$. In this range of $\theta$, a peak at the same frequency as that of the cross-flow force appears also in the drag signals; this feature is probably due to the large streamwise distance, for these configurations, between the two vertical edges where the vortices are generated, which implies a greater influence of one of the two shed vortices on the afterbody, and thus a significant fluctuation at the vortex shedding frequency also for the drag force.

The variation with aspect ratio and wind direction of the Strouhal number derived from the peaks of the cross-flow spectra for model 60 are shown in Fig. 13(a). As can be seen, St is found to increase with decreasing aspect ratio, particularly for the two lowest values. Actually, this feature is enhanced, for all aspect ratios, approaching the wind directions at which the flow reattachment on the leeward surface occurs. This result may be ascribed to the narrowing of the wake width occurring in proximity to the model free-end, due to the flow passing over the body; thus, this 3-D flow characteristic seems to produce an increasing effect on the average vortex shedding frequency as the aspect ratio of the model is decreased.

As for model 90, dominant spectral components in the fluctuations of the cross-flow force were singled out for two different ranges of wind direction: $0^{\circ} \leq \theta \leq 60^{\circ}$ and $100^{\circ} \leq \theta \leq 150^{\circ}$ (Fig. 13(b)). These configurations correspond to a separated flow over the leeward surface of the model and, thus, to the presence of a significant streamwise projection of the afterbody. In the lower range of $\theta$, the values of the Strouhal numbers for model 90 are of the same order as those of model 60, which could be expected, considering that for these wind directions the cross-flow widths of both models are comparable. Conversely, for the higher range of $\theta$ the Strouhal numbers for model 90 are much higher, due to the 


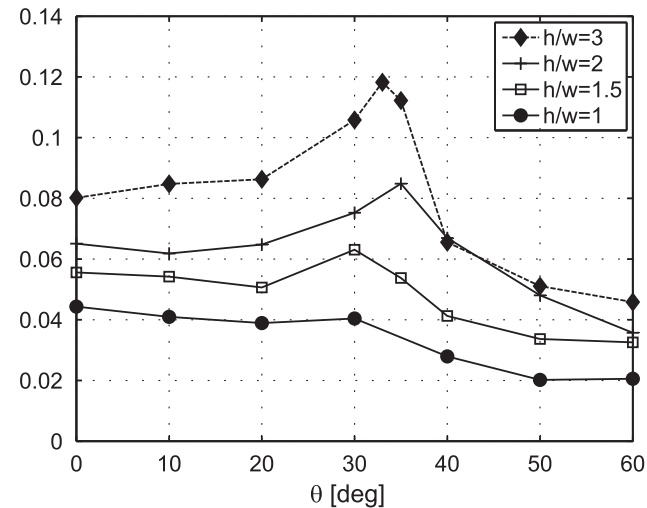

(a)

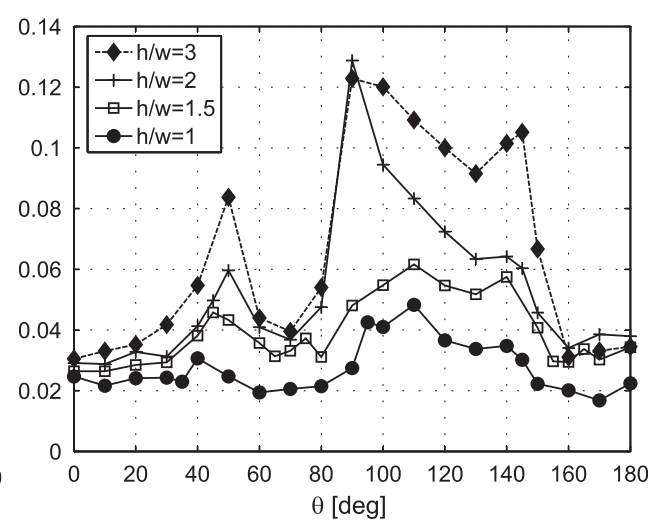

(b)

Fig. 14. Standard deviation of the cross-flow force coefficient as a function of wind direction and aspect ratio: (a) model 60 and (b) model 90 .

significantly smaller cross-flow width of this model for these wind directions. Finally, the trend of the Strouhal numbers shown in Fig. 13(b) is similar to the one obtained through hot-wire measurements by El-Sherbiny (1983), but with much lower values due to the flow three-dimensionality generated by the models with finite aspect ratio. Furthermore, it is seen that an increase in Strouhal number with reducing aspect ratio is also found for model 90 , and that this feature is enhanced by approaching wind orientations for which the reattachment of the flow on the leeward surface occurs.

Finally, the variations of the standard deviation values of the cross-flow force coefficient with wind direction and aspect ratio are shown in Figs. 14(a) and (b), respectively for model 60 and model 90. The first point that arises from the analysis of these figures is the significant reduction of the cross-flow force fluctuations with decreasing aspect ratio. This result might have been expected, as the effects of the flow passing over the free-end of the models become more and more important with decreasing aspect ratio, so that the influence of the alternate vortex shedding becomes less and less significant. As for the models with highest aspect ratio, it can be seen that the fluctuating forces are definitely higher for the wind orientations for which the flow is not attached over two faces of the models, with values that are roughly proportional to the longitudinal extent of the afterbody. Particularly significant is the fact that the maximum values are found for wind directions at which the flow is almost reattaching on the leeward face $\left(\theta \simeq 30^{\circ}\right.$ for model 60 and $\theta \simeq 50^{\circ}$ and $90^{\circ}$ for model 90 ). For these orientations not only a significant streamwise projection of the afterbody is present, but also one of the separated shear layers is at a minimum distance from the leeward surface.

\section{Conclusions}

Triangular prisms placed vertically on a plane, having isosceles cross-section with $60^{\circ}$ or $90^{\circ}$ apex angles and aspect ratio, $h / w$, ranging from 1.0 to 3.0 , were tested for wind directions, $\theta$, varying between $0^{\circ}$ and $180^{\circ}$ at a prevailing Reynolds number $\operatorname{Re}=w U_{\infty} / v \simeq 1.2 \times 10^{5}$.

Preliminary flow visualizations through tufts, performed only for model 60 with $h / w=3.0$, allowed the flow in proximity to the model to be characterized. Proceeding from the configuration with the wind directed against the apex edge of the prism $\left(\theta=60^{\circ}\right)$ to lower values of $\theta$, a flow recirculation region on the leeward vertical surface of the model in proximity to the front vertical edge was detected. This recirculation region increases its streamwise extent with reducing $\theta$, and at $\theta=35^{\circ}$ the flow can be considered as completely separated over the leeward vertical face of the prism.

Subsequently, hot-wire measurements were performed for the same model, in order to characterize the wake width and position. The vortex shedding frequency was singled out for each tested wind direction, and it was found to be roughly inversely proportional to the wake width evaluated through the maxima of the velocity fluctuations. However, a variation of the behaviour of the relevant Strouhal number was apparent for orientations for which two axial counterrotating vortices are present over the model free-end.

Force measurements carried out for all the models showed that the mean force coefficients decrease slightly with decreasing aspect ratio, and that for wind orientations at which the wake reattaches on the leeward vertical surface the absolute value of the mean cross-flow force may be definitely higher than the mean drag. Furthermore, the ratio 
between the mean drag forces acting on the prisms and those corresponding to 2-D bodies with the same cross-section was found to be dependent on the wind orientation, presumably in connection with differences in the free-end flow features.

The analysis of the fluctuating force signals permitted to ascertain that the vortex shedding frequency was present in the cross-flow force signals at all wind directions for which the streamwise projection of the portion of body surface immersed in the separated wake was significant. Furthermore, it was found that the vortex shedding frequency varies as a function of flow orientation in strict correlation with the variation of the width of the wake, and that it increases with decreasing aspect ratio. Finally, for the models with highest aspect ratio the standard deviations of the cross-flow force coefficients were found to be approximately proportional to the afterbody extent, but a significant reduction of the intensity of the fluctuations was found with decreasing aspect ratio.

\section{Acknowledgements}

The authors are grateful to G. Lombardi for his contribution in setting out the investigation, and to A. Agresta, M. Capurro and F. Schepis for their help in the execution of the tests. The financial support of the Italian Ministry of University and Research, M.U.R., and of the National Research Council, C.N.R., is also acknowledged.

\section{References}

Adamarola, M.S., Akinlade, O.G., Sumner, D., Bergstrom, D.J., Schenstead, A.J., 2006. Turbulent wake of a finite circular cylinder of small aspect ratio. Journal of Fluids and Structures 22, 919-928.

Afgan, I., Moulinec, C., Prosser, R., Laurence, D., 2007. Large eddy simulation of turbulent flow for wall mounted cantilever cylinders of aspect ratio 6 and 10. International Journal of Heat and Fluid Flow 28, 561-574.

Alonso, G., 2005. Fenomenos de galope en obstaculos de section no rectangular. Ph.D. Thesis, Universidad Politecnica de Madrid.

Ayoub, A., Karamcheti, K., 1982. An experiment on the flow past a finite circular-cylinder at high subcritical and supercritical Reynolds-numbers. Journal of Fluid Mechanics 118, 1-26.

Budair, M., Ayoub, A., Karamcheti, K., 1991. Frequency measurements in a finite cylinder wake at a subcritical Reynolds-number. AIAA Journal 29 (12), 2163-2168.

Buresti, G., Iungo, G.V., 2008. Flow fluctuations and vorticity dynamics in the near-wake of a triangular prism in cross-flow. In: Proceedings of International Colloquium on Bluff Body Aerodynamics and Applications, BBAA VI, Milano, July $20-24$.

Buresti, G., Lombardi, G., 2000. Application of wavelet transform to the analysis of velocity fluctuations in the wake of triangular prisms. In: Proceedings of the Sixth Italian Congress on Wind Engineering, In-Vento, pp. 301-308.

Buresti, G., Lombardi, G., Bellazzini, J., 2004. On the analysis of fluctuating velocity signals through methods based on the wavelet and Hilbert transforms. Chaos, Solitons \& Fractals 20 (1), 149-158.

Buresti, G., Lombardi, G., Talamelli, A., 1998. Low aspect-ratio triangular prisms in cross-flow: measurements of the wake fluctuating velocity field. Journal of Wind Engineering and Industrial Aerodynamics 74-76, 463-473.

Camarri, S., Salvetti, M.V., Buresti, G., 2006. Large-eddy simulation of the flow around a triangular prism with moderate aspect-ratio. Journal of Wind Engineering and Industrial Aerodynamics 94 (5), 309-322.

El-Sherbiny, S., 1983. Flow separation and reattachment over the side of a $90^{\circ}$ triangular prism. Journal of Wind Engineering and Industrial Aerodynamics 11, 393-403.

E.S.D.U., 1979. Mean fluid forces and moments on cylindrical structures: polygonal sections with rounded corners including elliptic shapes. E.S.D.U. (Item 79026).

Farivar, D.J., 1981. Turbulent uniform-flow around cylinders of finite length. AIAA Journal 19 (3), $275-281$.

Fox, T.A., Apelt, C.J., West, G.S., 1993. The aerodynamic disturbance caused by the free-ends of a circular-cylinder immersed in a uniform-flow. Journal of Wind Engineering and Industrial Aerodynamics 49 (1-3), 389-399.

Fröhlich, J., Rodi, W., 2004. LES of the flow around a circular cylinder of finite height. International Journal of Heat and Fluid Flow $25,537-548$.

Iungo, G.V., Buresti, G., 2007. Experimental investigation on the wake generated from a low aspect-ratio triangular prism in crossflow. N. ADIA 2007-4, Atti del Dipartimento di Ingegneria Aerospaziale dell'Università di Pisa, ETS Editrice, Pisa.

Kitagawa, T., Fujino, Y., Kimura, Y., Mizuno, Y., 2002. Wind pressures measurement on end-cell-induced vibration of a cantilevered circular cylinder. Journal of Wind Engineering and Industrial Aerodynamics 90, 395-405.

Kitagawa, T., Wakahara, T., Fujino, Y., Kimura, Y., 1997. An experimental study on vortex-induced vibration of a circular cylinder tower at a high wind speed. Journal of Wind Engineering and Industrial Aerodynamics 71, 731-744.

Lindsey, W.F., 1938. Drag of cylinders of simple shapes. NACA Report 619.

Liu, Y., So, R.M.C., Cui, Z.X., 2005. A finite cantilevered cylinder in a cross-flow. Journal of Fluids and Structures 20 , 589-609.

Luo, S.C., Yazdani, Md.G., Chew, Y.T., Lee, T.S., 1994. Effects of incidence and afterbody shape on flow past bluff cylinders. Journal of Wind Engineering and Industrial Aerodynamics 53, 375-399. 
Okamoto, S., Sunabashiri, Y., 1992. Vortex shedding from a circular-cylinder of finite length placed on a ground plane. ASME Journal of Fluids Engineering 114 (4), 512-521.

Park, C.W., Lee, S.J., 2000. Free end effects on the near wake flow structure behind a finite circular cylinder. Journal of Wind Engineering and Industrial Aerodynamics 88 (2-3), 231-246.

Pattenden, R.J., Turnock, S.R., Zhang, X., 2005. Measurements of the flow over a low-aspect-ratio cylinder mounted on a ground plane. Experiments in Fluids 39 (1), 10-21.

Roh, S.C., Park, S.O., 2003. Vortical flow over the free end surface of a finite circular cylinder mounted on a flat plate. Experiments in Fluids 34, 63-67.

Sakamoto, H., Arie, M., 1983a. Flow around a normal plate of finite width immersed in a turbulent boundary-layer. ASME Journal of Fluids Engineering 105 (1), 98-104.

Sakamoto, H., Arie, M., 1983b. Vortex shedding from a rectangular prism and a circular-cylinder placed vertically in a turbulent boundary-layer. Journal of Fluid Mechanics 126, 147-165.

Srigrarom, S., Koh, A.K.G., 2008. Flow field of self-excited rotationally oscillating equilateral triangular cylinder. Journal of Fluids and Structures 24, 750-755.

Sumner, D., Heseltine, J.L., Dansereau, O.J.P., 2004. Wake structure of a finite circular cylinder of small aspect ratio. Experiments in Fluids 37, 720-730. 